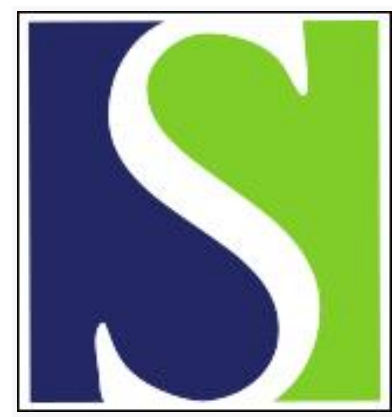

Scand J Work Environ Health 2002;28(5):293-303

https://doi.org/10.5271/sjweh.678

Issue date: Oct 2002

Low-back and shoulder complaints among workers with pushing and pulling tasks

by Hoozemans MJM, van der Beek AJ, Frings-Dresen MHW, van der Woude LHV, van Dijk FJH

Affiliation: Coronel Institute for Occupational and Environmental Health, AmCOGG Amsterdam Center for Research into Health and Health Care, Academic Medical Center / University of Amsterdam, Amsterdam, Netherlands.

Refers to the following texts of the Journal: $1999 ; 25$ suppl $4: 36-42$ 1996;22(4):251-259 1997;23(5):370-377 1996;22(2):94-101 2001;27 suppl 1:1-102 2001;27(2):125-132 1999;25 suppl 1:31-33 $1997 ; 23(4): 243-256 \quad 1999 ; 25(5): 387-403$

The following articles refer to this text: 2002;28(5):289-292; 2004;30(4):279-286

Key terms: longitudinal study; low-back complaint; low-back pain; manual materials handling; observation; occupational exposure; prospective study; pulling task; pushing task; questionnaire; regression analysis; shoulder complaint; shoulder pain; worker

This article in PubMed: www.ncbi.nlm.nih.gov/pubmed/12432982

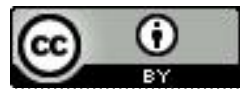




\title{
Low-back and shoulder complaints among workers with pushing and pulling tasks
}

\author{
by Marco JM Hoozemans, PhD, ${ }^{1}$ Allard J van der Beek, PhD, ${ }^{1,2}$ Monique HW Frings-Dresen, PhD, ${ }^{1}$ \\ Luc HV van der Woude, $P h D^{3}$ Frank JH van Dijk, $P h D^{1}$
}

\begin{abstract}
Hoozemans MJM, van der Beek AJ, Frings-Dresen MHW, van der Woude LHV, van Dijk FJH. Low-back and shoulder complaints among workers with pushing and pulling tasks. Scand $J$ Work Environ Health 2002;28(5):293-303.
\end{abstract}

Objectives Low-back and shoulder complaints were examined in relation to self-reported and objectively assessed exposure to work-related pushing and pulling.

Methods Workers from several companies (eg, nursing homes and flower auctions) with pushing and pulling tasks and, as reference, workers without physically demanding tasks were invited to participate. Altogether 829 workers initially received a questionnaire, of whom 459 responded both initially and after 1 year of follow-up. Initially, self-reported exposure to pushing and pulling was assessed by questionnaire. Furthermore, a representative sample of 131 workers was observed at work using TRAC (task recording and analysis on computer) to assess exposure to pushing and pulling objectively. For exposure to pushing and pulling, the workers were classified into a reference group and medium and high exposure groups. Initially and in the follow-up, the 12month prevalence of low-back and shoulder complaints was assessed. Complaints reported in the follow-up were separately used as dependent variables to calculate prevalence rate ratios (PR) in a Cox's proportional hazard regression analysis.

Results The adjusted PR values were not significant for low-back complaints. For shoulder complaints, both the medium and high exposure groups showed significant adjusted PR values for self-reported exposure and observed duration of pushing and pulling when compared with the reference group (PR range 2.18-4.86). For the observed frequency of pushing and pulling, only the medium exposure group showed a significant PR, of 3.95.

Conclusions The findings suggest a rather strong relationship between pushing and pulling and shoulder complaints. The evidence for a relationship between pushing and pulling and low-back complaints is limited.

Key terms longitudinal study, low-back pain, materials handling, observation, occupational exposure, prospective study, questionnaire, regression analysis, shoulder pain.

Manual handling of materials is a daily routine activity for a substantial number of workers. Generally, these workers run a considerable risk of developing musculoskeletal complaints. Recent reviews of the epidemiologic literature indicate a relation between manual materials handling and low-back complaints $(1,2)$. For shoulder complaints the association is less consistent $(3$,
4). It can be argued whether these associations apply to both the lifting and carrying and the pushing and pulling of loads, which are estimated to be equally frequent techniques in manual materials handling (5) but which are essentially different from a mechanical perspective. Lifting loads has been extensively studied and was found to be consistently related to low-back complaints

1 Coronel Institute for Occupational and Environmental Health, AmCOGG Amsterdam Center for Research into Health and Health Care, Academic Medical Center / University of Amsterdam, Amsterdam, The Netherlands.

2 Department of Social Medicine and Institute for Research in Extramural Medicine, VU University Medical Center, Amsterdam, The Netherlands.

3 Institute for Fundamental and Clinical Human Movement Sciences, Faculty of Human Movement Sciences, Vrije Universiteit, Amsterdam, The Netherlands.

Reprint requests to: Dr Monique HW Frings-Dresen, Coronel Institute for Occupational and Environmental Health, Academic Medical Center / University of Amsterdam, PO Box 22700, NL-1100 DE Amsterdam, The Netherlands. [E-mail: m.frings@amc.uva.nl] 
(6). The risk of musculoskeletal complaints related to pushing and pulling is less well documented (7). Of all back strain and pain, $9-18 \%$ was found to be associated with pushing and pulling $(8,9)$. Several other crosssectional studies have reported an association between pushing and pulling and low-back complaints (10-14). An association between pushing and pulling and shoulder complaints has been reported by van der Beek et al (13) and Hughes et al (15) only. Thus the evidence for a (causal) relationship between pushing and pulling and low-back or shoulder complaints is not conclusive, mainly because only cross-sectional designs have been used.

Prospective study designs are less susceptible to bias than with cross-sectional designs and can be considered the next step in identifying pushing and pulling as a risk factor for musculoskeletal complaints $(7,16)$. The causality of the relation can be further explored by establishing an exposure-response relationship. This may also provide a scientific basis for the development of ergonomic adjustments of pushing and pulling tasks to prevent musculoskeletal complaints. To this end, an adequate assessment of exposure is essential $(17,18)$. Exposure is usually defined by job title only or by using self-administered questionnaires. However, self-reported exposure may result in misclassification of exposure and an attenuation of the association with musculoskeletal complaints in case the misclassification is nondifferential (19). Hence exposure to pushing and pulling should be assessed at a sufficient level of detail, with the use of objective measurement methods where possible (18).

The current prospective cohort study was performed among workers with mainly work-related pushing and pulling tasks and a reference group that was not involved in physically demanding work. The objective was to study low-back and shoulder complaints in relation to self-reported and objectively assessed exposure to pushing and pulling.

\section{Subjects and methods}

\section{Design and study population}

A 1-year prospective cohort study of male and female workers was initiated in 1997. Relatively large companies and institutions, for which it was known that pushing and pulling were common activities, were approached to participate in the study. Companies were selected for participation after approval of the management and after a visual inspection at the workplace to insure that pushing and pulling were prominent activities. Eventually, a rail catering company that provides food and drinks in trains, two nursing homes, two flower auctions, a distribution center of the Dutch postal services, and a large group of refuse collectors from several small companies were included in the study. (See also the appendix.) Workers were invited to participate only if they held a permanent job. Furthermore, within each company, two groups of workers were included. First, workers who regularly performed pushing and pulling tasks were included. Second, to serve as reference, workers who performed tasks that were not physically demanding, such as administrative or management tasks, were included.

All of the participants were requested to complete a questionnaire twice, with a follow-up period of 1 year. The first questionnaire was presented to 829 employees. If the response rate was low, the employees were approached at work, and in some cases they were assisted in completing the questionnaire. Initially, 629 (76\%) questionnaires were completed. At the time of the follow-up, 38 participants were no longer employed by the company and were not available. Of the remaining 591 participants, 459 (78\%) returned the follow-up questionnaire. (Detailed information is also presented in the appendix.)

\section{Assessment of independent variables}

Exposure to pushing and pulling was subjectively assessed initially using the Dutch musculoskeletal questionnaire (DMQ) (20-22), in which the question was asked whether pushing or pulling a total weight over 50 kilograms was a frequent activity. The answers were assessed on a 4-point scale (seldom or never, sometimes, often, very often). For each exposed and reference group within each company the percentage of workers scoring within a category of the 4-point scale was calculated. The percentage per category was multiplied by 1 for the seldom or never category to 4 for the very often category. Hence, a score of 100 was awarded to the exposed and reference groups within each company if they had a $100 \%$ score within seldom or never, and a score of 400 was given to groups with a $100 \%$ score within the category very often. Next, the exposed and reference groups within each company were categorized into three exposure groups. The initially selected groups of workers without physically demanding tasks, included as the reference group, had a score below 200 points. Furthermore, the groups of workers exposed to pushing and pulling were categorized into a medium exposure group (200-299 points) and a high exposure group (300-400 points). For detailed information concerning the categorization of the exposed and reference groups within each company the reader is referred to the appendix.

A representative sample of workers from each company was also systematically observed initially at their 
workplace using TRAC (task recording and analysis on computer) software (23). Of the workers exposed to pushing and pulling, 92 were observed [68 men, 24 women, mean age 38.1 years (SD 10.1)]. Of the reference group 39 workers were observed [ 21 men, 18 women, mean age 38.8 years (SD 9.7)]. Each selected worker exposed to pushing and pulling was followed by two observers for approximately a full workday. The workers in the reference group were observed for half a day. Preceding the observations, the participating observers were trained to improve inter- and intraobserver reliability. During a week of intense training, the percentage of agreement and the Cohen's kappa between and within observers were assessed for all the variables. At the end of the training period, it was insured that all the variables had a percentage of agreement of at least $80 \%$ and a Cohen's kappa of at least 0.50 , which is acknowledged as an acceptable standard of observer reliability (24). The tasks, activities, and materials handled were observed continuously by one of the observers. Activities were divided into lifting, carrying, pushing, pulling, standing, sitting, walking, and kneeling. Work postures (ie, trunk flexion, trunk rotation and lateroflexion, and arm elevation) were recorded every 15 seconds by the other observer. For each of the exposed and reference groups within each company the average exposure to activities and postures was calculated using the observation data and company specific information such as work schedules and job rotation. A group strategy was used [ie, each worker in the exposed and reference groups within each company was modeled to have the same exposure, which was defined as the daily frequency (times per day) of activities and the duration (seconds per day) of activities and work postures]. For the frequency, as well as the duration, the results for push- ing were added to those for pulling and categorized into a reference group, medium exposure group, and high exposure group. Again, the reference group consisted of the original reference population. To classify the medium and high exposure groups, a cut-off point was chosen based on a natural boundary (ie, the contrast in the observed frequency or duration between two successive companies had to be relatively large) and on a relatively equal division in the number of workers. The demographic characteristics of the exposure groups are presented in table 1 according to the self-reported exposure to pushing and pulling and on the observed frequency and duration of pushing and pulling.

Recent reviews on risk factors for low-back and shoulder complaints were screened for potential confounders or effect modifiers in the association between pushing and pulling and low-back or shoulder complaints (1-4, 6, 25-28). Working behind a computer display, driving vehicles, lifting or carrying loads, trunk flexion (>20 degrees), trunk rotation and lateroflexion, and elevation of one or both arms (>60 degrees) were assessed by observation at the workplace and considered to be potential confounders. The work postures were quantified as the daily time spent in these postures. The daily time spent in these postures while pushing, pulling, or lifting was subtracted from the total time in the work postures to estimate eventually the effect of posture independently of pushing, pulling, or lifting. For the analyses, these variables were dichotomized into low and high exposure. Again, cut-off points were chosen based on a relatively large natural boundary between the frequencies of the concerning variable of successive companies and on a relatively equal division in the number of workers. Gender, age, level of education (as indicator of socioeconomic status), and years of

Table 1. Number of respondents (male and female) in both the baseline examination and the 1-year follow-up of the questionnaire survey, age, employment in current job, and the number of workhours for the three exposure groups of self-reported exposure to pushing and pulling and the observed frequency and duration of pushing and pulling.

\begin{tabular}{|c|c|c|c|c|c|c|c|c|}
\hline \multirow[t]{2}{*}{ Pulling and pushing } & \multirow{2}{*}{$\begin{array}{l}\text { Men } \\
(\mathrm{N})\end{array}$} & \multirow{2}{*}{$\begin{array}{l}\text { Women } \\
\text { (N) }\end{array}$} & \multicolumn{2}{|c|}{ Age (years) } & \multicolumn{2}{|c|}{ Employment in current job (years) } & \multicolumn{2}{|c|}{ Workhours per wed } \\
\hline & & & Mean & SD & Mean & $\mathrm{SD}$ & Mean & SD \\
\hline \multicolumn{9}{|l|}{ Self-reported exposure } \\
\hline Reference group & 64 & 76 & 41.6 & 8.8 & 11.9 & 6.9 & 34 & 10 \\
\hline Medium exposure group & 91 & 106 & 39.3 & 9.3 & 9.6 & 6.7 & 33 & 11 \\
\hline High exposure group & 102 & 11 & 41.3 & 8.8 & 13.9 & 8.0 & 38 & 3 \\
\hline \multicolumn{9}{|l|}{ Observed frequency } \\
\hline Reference group & 64 & 76 & 41.6 & 8.8 & 11.9 & 6.9 & 34 & 10 \\
\hline Medium exposure group & 46 & 109 & 40.1 & 9.2 & 11.2 & 7.1 & 31 & 9 \\
\hline High exposure group & 147 & 8 & 40.0 & 9.1 & 11.2 & 7.8 & 38 & 9 \\
\hline \multicolumn{9}{|l|}{ Observed duration } \\
\hline Reference group & 64 & 76 & 41.6 & 8.8 & 11.9 & 6.9 & 34 & 10 \\
\hline Medium exposure group & 70 & 100 & 39.7 & 9.3 & 10.1 & 6.6 & 34 & 10 \\
\hline High exposure group & 123 & 17 & 40.5 & 9.0 & 12.5 & 8.2 & 36 & 8 \\
\hline
\end{tabular}


employment were individually assessed using the baseline questionnaire. In addition, the psychosocial factors decision authority, skill discretion, psychosocial work demands, co-worker support, and supervisor support were assessed using the questionnaire on perception and the judgment of work (29). The individual's exposure to the psychosocial factors was dichotomized into low and high exposure using the population's median level as the cut-off point.

\section{Assessment of dependent variables}

Initially and at the follow-up the participants were requested to complete a Dutch translation of the Standardized Nordic Questionnaire (30). The question of whether the participant had experienced trouble (ache, pain, discomfort) during the last 12 months was used to determine the 12-month prevalence of complaints. The 12-month prevalences of low-back and shoulder complaints reported at the time of the follow-up were separately used as dependent variables in the analyses.

\section{Analyses and statistics}

Prevalence rate ratios (PR) and corresponding 95\% confidence intervals $(95 \% \mathrm{CI}$ ) were calculated using Cox's proportional hazards regression analysis with a constant risk period to study the relation between pushing and pulling and the 12-month prevalence of low-back and shoulder complaints at the time of the follow-up. The crude prevalence rate ratios between the groups with medium and high exposure to pushing and pulling and the reference group were calculated for the self-reported exposure and the observed frequency and duration of pushing and pulling.

With the use of multivariate analyses, prevalence rate ratios were adjusted for confounding and effect modification. To prevent collinearity, correlation coefficients between the independent variables were calculated. Working behind a computer display was highly (negatively) correlated with all the exposure measures of pushing and pulling with absolute correlation coefficients above 0.8 . Driving vehicles was highly (positively) correlated with the observed frequency of pushing and pulling. These variables were subsequently omitted from the epidemiologic models. Each of the remaining independent variables was screened separately for confounding. The prevalence rate ratios were a priori adjusted for gender and age, with age defined as a continuous variable (years). If the prevalence rate ratio for pushing and pulling, adjusted for gender and age, changed more than $10 \%$, the included variable was identified as a confounder and included in the multivariate model. Only carrying loads and educational level appeared to affect the prevalence rate ratios for lowback complaints and shoulder complaints, respectively.

The 12-month prevalences of low-back and shoulder complaints reported initially were also examined for confounding and effect modification. For all the measures of exposure, the complaints reported initially affected the prevalence rate ratios for complaints reported at the time of the follow-up. Interaction between complaints reported initially and exposure to pushing and pulling was separately examined for effect modification and was significant in all the analyses with shoulder complaints. Therefore, it was decided to include both the initial complaints and the interaction with exposure to pushing and pulling in the multivariate model, for both low-back and shoulder complaints, to estimate the contribution of exposure to pushing and pulling to complaints reported at the time of the follow-up controlled for complaints reported initially.

All the statistical analyses were performed with version 9.0.0 of the SPSS (Statistical Package for the Social Sciences), and a significance level of $5 \%$ was used.

\section{Nonresponse analysis}

Of the 459 participants who returned the follow-up questionnaire, 11 retired or became unemployed. Multivariate nonresponse analyses were used to identify factors associated with losses to follow-up (31). Generally, the female participants and participants who were employed for a longer period of time were more likely to respond. The 12-month prevalences of both low-back and shoulder complaints at the beginning of the study were not associated with losses to follow-up.

\section{Results}

\section{Observed exposure to pushing and pulling}

Table 2 presents the mean and range for the observed frequency (times per day) and duration (seconds per day) of pushing and pulling, categorized into three exposure groups. The highest values for frequency, up to an average of 1000 pushing and pulling actions per day, were observed for the refuse collectors and workers of the postal distribution center. Workers of the postal distribution center and the rail catering company pushed and pulled for the largest part of the workday (about $20 \%$ ). For detailed information at the company level the reader is referred to the appendix.

\section{Pushing and pulling in relation to low-back complaints}

Table 3 presents the crude and adjusted prevalence rate ratios for the 12-month prevalence of low-back 
complaints at the time of the follow-up. With respect to the observed frequency, a significantly increased crude prevalence rate ratio was found only for the medium exposure group (PR 1.39, 95\% CI 1.00-1.94). For the observed duration of pushing and pulling, the medium exposure group had a marginally significant crude prevalence rate ratio (PR 1.36, 95\% CI 0.98-1.89). None of the adjusted prevalence rate ratios for the low-back complaints at the time of follow-were significantly increased. Of the confounding factors, the 12-month prevalence of low-back complaints at the beginning of the study had a significantly increased prevalence rate ratio of nearly 4 for low-back complaints at the time of follow-up for all the definitions of exposure.

\section{Pushing and pulling in relation to shoulder complaints}

The crude prevalence rate ratios for shoulder complaints were significantly increased for self-reported exposure in the high exposure group and for the observed frequency and duration in the medium exposure groups (table 4). The adjusted prevalence rate ratios were significantly increased for both the medium and high exposure groups with respect to the self-reported exposure to pushing and pulling, with prevalence rate ratios of 2.90 and 4.86, respectively. For the observed frequency, only the medium exposure group had a significantly increased prev- alence rate ratio (PR 3.95, 95\% CI 1.56-9.96). For the observed duration, both the medium and high exposure groups had a significantly increased adjusted prevalence rate ratio. The prevalence rate ratio for the medium exposure group was 3.33 (95\% CI 1.32-8.36), and for the high exposure group it was 2.67 (95\% CI 1.01-7.07). Furthermore, the effect of including shoulder complaints at the beginning of the study in the multivariate model was substantial. Both confounding and effect modification appeared to be present. For the present analyses, therefore, the presented prevalence rate ratios for exposure to pushing and pulling are valid for the participants initially without shoulder complaints. For the participants with shoulder complaints initially, the risk of pushing and pulling differs and can be calculated by

Table 2. Results of the observations of workers at their workplace in terms of the mean and range of frequency (times per day) and duration (seconds per day) of pushing and pulling.

\begin{tabular}{lcccccc}
\hline Group & \multicolumn{2}{c}{ Observed frequency } & & \multicolumn{2}{c}{ Observed duration } \\
\cline { 2 - 3 } \cline { 6 - 7 } & $\begin{array}{l}\text { Mean (times/ } \\
\text { day) }\end{array}$ & Range & & $\begin{array}{l}\text { Mean (seconds/ } \\
\text { day) }\end{array}$ & Range \\
\hline Reference & 16 & $0-44$ & & 131 & $0-310$ \\
Medium exposure & 135 & $88-234$ & & 1344 & $783-2599$ \\
High exposure & 741 & $464-982$ & & 4139 & $3520-5098$ \\
\hline
\end{tabular}

Table 3. Relation between low-back complaints and exposure to pushing and pulling. Confounding factors and effect modifiers with corresponding prevalence rate ratios are presented when included in the regression model. Gender and age were forced into the model a priori. Age was entered into the model as a continuous variable. ( $\mathrm{PR}=$ prevalence rate ratio, $\mathrm{Adj}=$ adjusted, $95 \% \mathrm{Cl}=95 \%$ confidence interval)

\begin{tabular}{|c|c|c|c|c|c|c|c|c|c|c|c|}
\hline & \multicolumn{4}{|c|}{ Self-reported exposure } & \multicolumn{4}{|c|}{ Observed frequency } & \multicolumn{3}{|c|}{ Observed duration } \\
\hline & Orude P & $\mathrm{R} 95 \% \mathrm{C}$ & Adj PR & $95 \% \mathrm{Cl}$ & Crude PI & $\mathrm{R} 95 \% \mathrm{Cl}$ & Adj PR & $95 \% \mathrm{Cl}$ & Orude PR & $95 \% \mathrm{Cl}$ & Adj PR $95 \% \mathrm{Cl}$ \\
\hline \multicolumn{12}{|l|}{ Group } \\
\hline Reference & 1.00 & . & 1.00 & . & 1.00 & . & 1.00 & . & 1.00 & . & 1.00 \\
\hline Medium exposure & 1.22 & $0.88-1.68$ & 1.53 & $0.76-3.09$ & $9 \quad 1.39$ & $1.00-1.94$ & 1.72 & $0.81-3.62$ & 21.36 & $0.98-1.89$ & $1.23 \quad 0.57-2.63$ \\
\hline High exposure & 1.29 & $0.90-1.84$ & 41.74 & $0.82-3.70$ & 1.10 & $0.78-1.55$ & 1.52 & $0.74-3.08$ & 81.10 & $0.77-1.57$ & $1.97 \quad 0.97-3.98$ \\
\hline \multicolumn{12}{|l|}{ Confounding factors } \\
\hline \multicolumn{12}{|l|}{ Gender } \\
\hline Men & . & . & 1.00 & & . & . & 1.00 & & . & . & 1.00 \\
\hline Women & . & . & 1.020 & $0.75-1.38$ & . & . & 0.93 & $0.66-1.29$ & . & . & $1.020 .75-1.37$ \\
\hline Age (years) & . & . & 0.990 & $0.98-1.01$ & . & . & 0.99 & $0.98-1.01$ & . & . & $0.990 .98-1.01$ \\
\hline \multicolumn{12}{|l|}{ Carrying loads } \\
\hline Group with low exposure & . & . & 1.00 & & . & . & 1.00 & & . & . & 1.00 \\
\hline Group with high exposure & . & . & 1.260 & $0.86-1.85$ & . & . & 1.34 & $0.92-1.97$ & . & . & $1.260 .88-1.81$ \\
\hline \multicolumn{12}{|l|}{ Low-back complaints at baseline } \\
\hline No low-back complaints & . & . & 1.00 & & . & . & 1.00 & & . & . & 1.00 \\
\hline Low-back complaints & . & . & 3.852 & $2.04-7.28$ & . & . & 3.86 & $2.04-7.30$ & . & . & $3.852 .03-7.28$ \\
\hline \multicolumn{12}{|l|}{ Effect modification } \\
\hline \multicolumn{12}{|l|}{$\begin{array}{l}\text { Exposure } \times \text { low-back complaints } \\
\text { in the baseline survey }\end{array}$} \\
\hline $\begin{array}{l}\text { Medium exposure } \times \text { low-back } \\
\text { complaints }\end{array}$ & & . & 0.810 & $0.37-1.78$ & & . & 0.79 & $0.34-1.80$ & . & . & $1.060 .46-2.47$ \\
\hline $\begin{array}{l}\text { High exposure } \times \text { low-back } \\
\text { complaints }\end{array}$ & . & . & 0.700 & $0.30-1.63$ & & . & 0.72 & $0.32-1.61$ & . & . & $0.550 .25-1.25$ \\
\hline
\end{tabular}


Table 4. Relation between shoulder complaints and exposure to pushing and pulling. Confounding factors and effect modifiers with corresponding prevalence rate ratios are presented when included in the regression model. Gender and age were forced into the model a priori. Age was entered into the model as a continuous variable. ( $P R=$ prevalence rate ratio, $\mathrm{Adj}=$ adjusted, $95 \% \mathrm{Cl}=95 \%$ confidence interval)

\begin{tabular}{|c|c|c|c|c|c|c|c|c|c|c|c|c|}
\hline & \multicolumn{4}{|c|}{ Self-reported exposure } & \multicolumn{4}{|c|}{ Observed frequency } & \multicolumn{4}{|c|}{ Observed Duration } \\
\hline & Crude PR & $\mathrm{R} 95 \% \mathrm{Cl}$ & Adj PR & $95 \% \mathrm{Cl}$ & Crude P & $\mathrm{R} 95 \% \mathrm{Cl}$ & Adj PR & $95 \% \mathrm{Cl}$ & Crude PF & $\mathrm{R} 95 \% \mathrm{Cl}$ & Adj PR & $95 \% \mathrm{Cl}$ \\
\hline \multicolumn{13}{|l|}{ Group } \\
\hline Reference & 1.00 & . & 1.00 & . & 1.00 & . & 1.00 & . & 1.00 & . & 1.00 & . \\
\hline Medium exposure & 1.40 & $0.90-2.17$ & 2.90 & $1.17-7.16$ & 2.05 & $1.33-3.16$ & 3.95 & $1.56-9.96$ & 1.89 & $1.23-2.91$ & 3.33 & $1.32-8.36$ \\
\hline High exposure & 2.20 & $1.41-3.45$ & 4.86 & $1.85-12.79$ & 1.36 & $0.85-2.15$ & 2.18 & $0.82-5.79$ & 1.47 & $0.92-2.33$ & 2.67 & $1.01-7.07$ \\
\hline \multicolumn{13}{|l|}{ Confounding factors } \\
\hline \multicolumn{13}{|l|}{ Gender } \\
\hline Men & . & . & 1.00 & & . & . & 1.00 & & . & . & 1.00 & \\
\hline Women & . & . & 1.26 & $0.85-1.89$ & . & . & 0.92 & $0.60-1.41$ & 1 . & . & 1.05 & $0.71-1.55$ \\
\hline Age (years) & & & 1.00 & $0.98-1.02$ & . & . & 1.00 & $0.98-1.02$ & 2 . & . & 1.00 & $0.98-1.02$ \\
\hline \multicolumn{13}{|l|}{ Education } \\
\hline High & . & . & - & . & . & . & 1.00 & & . & . & 1.00 & \\
\hline Low & . & . & - & . & . & . & 1.21 & $0.83-1.77$ & 7 . & . & 1.16 & $0.80-1.68$ \\
\hline \multicolumn{13}{|l|}{ Shoulder complaints at baseline } \\
\hline No shoulder complaints & . & . & 1.00 & & . & . & 1.00 & & . & . & 1.00 & \\
\hline Shoulder complaints & . & . & 10.79 & $4.39-26.55$ & . & . & 11.38 & $34.61-28.08$ & 3. & . & 11.04 & $44.48-27.22$ \\
\hline \multicolumn{13}{|l|}{ Effect modification } \\
\hline $\begin{array}{l}\text { Exposurex shoulder complaints } \\
\text { at baseline }\end{array}$ & . & . & · & . & . & . & . & . & . & . & . & . \\
\hline $\begin{array}{l}\text { Medium exposure } \times \text { shoulder } \\
\text { complaints }\end{array}$ & er. & . & 0.31 & $0.11-0.89$ & . & . & 0.26 & $0.09-0.75$ & 5 . & . & 0.30 & $0.11-0.87$ \\
\hline $\begin{array}{l}\text { High exposure } \times \text { shoulder } \\
\text { complaints }\end{array}$ & . & . & 0.26 & $0.09-0.77$ & . & . & 0.37 & $0.13-1.10$ & . & . & 0.35 & $0.12-1.04$ \\
\hline
\end{tabular}

multiplying the prevalence rate ratio for pushing and pulling $\times$ the prevalence rate ratio for effect modification. For instance, for the observed frequency of pushing and pulling the participants of the medium exposure group without shoulder complaints initially had a prevalence rate ratio of 3.95 . However, the participants of the medium exposure group who reported shoulder complaints initially had a prevalence rate ratio of 1.03 (3.95 $\times 0.26$ ). For all the exposure groups and all the exposure measures the prevalence rate ratios changed to values between 0.81 and 1.26 when the participants initially reported shoulder complaints. However, these participants were found to have a prevalence rate ratio of 11.38 for reporting shoulder complaints again in the 1-year follow-up when compared with the participants who did not report shoulder complaints initially.

\section{Discussion}

The objective of the present longitudinal study was to examine the relations of subjectively and objectively assessed exposure to pushing and pulling to low-back and shoulder complaints after 1 year. Significant prevalence rate ratios for exposure to pushing and pulling in relation to low-back complaints were not found. However, pushing and pulling appeared to be significantly related to shoulder complaints.

To our knowledge, the relation between pushing and pulling and musculoskeletal complaints has not been explored longitudinally. Significant associations reported in cross-sectional studies mainly point to the lowback region as being at risk (10-13). This finding could only tentatively be confirmed by our study. Hughes et al (15) found no association with lowback complaints, but they demonstrated an association with shoulder complaints in an univariate analysis. van der Beek et al (13) reported a significant odds ratio of 2.0 (90\% CI 1.1-3.7) for shoulder complaints among truck drivers who frequently pushed and pulled wheeled cages in comparison with truck drivers who performed hardly any loading or unloading activities. The prevalence rate ratios found by us were substantially higher. The characteristics of the population under study may in part explain the differences in the results. A relatively large contrast in exposure to pushing and pulling between the exposure and reference groups was obtained through the selection of the companies and workers (table 2). This contrast may reveal the specific risk of pushing and pulling, also because significant other risk factors for low-back and shoulder complaints, 
such as lifting and carrying, were rarely present or were even absent.

One of the work-related risk factors for shoulder complaints, working behind a computer display, appeared to be highly correlated with exposure (ie, workers who push and pull do not work behind computer displays). Because of this high correlation with exposure, working behind a computer display could not be entered into the regression model to account for the potential confounding effect of this factor. As a result, prevalence rate ratios for shoulder complaints could even be underestimated considering the fact that the prevalence of shoulder complaints may have been relatively increased in the reference group because of work behind computer displays (3). The prevalence of complaints in the reference group may also have been relatively high because of the relocation of workers who have developed complaints from jobs that are physically demanding to jobs without any physical activities.

The reference group showed somewhat lower prevalences at the time of the follow-up when compared with the initial values for both low-back and shoulder complaints. This finding may be the result of a tendency to overreport complaints in an earlier stage in the first questionnaire or to an unmotivated completion of the second questionnaire (test effect). It is expected that such occurrences would not affect the prevalence rate ratios because this type of bias can be considered nondifferential. Considering the present attention to the design of work environments, another explanation may be that ergonomic interventions by companies have proved to be effective. Unfortunately, none of these hypotheses could be confirmed by the results of our questionnaire survey.

Selection of the study population was further studied in nonresponse analyses. Unfortunately, the characteristics of the workers who did not respond to the first questionnaire could not be retrieved and may have biased the selection of the population. At the time of the follow-up, workers who did not respond did not substantially differ from the respondents. The 12-month prevalences of both low-back and shoulder complaints were not associated with losses to follow-up. However, the study population can be considered relatively old (about 40 years on the average) and workers employed for a longer period of time are more likely to respond at follow-up. Furthermore, the highest prevalence rate ratios for shoulder complaints (table 4) were observed in the medium exposure groups. An explanation for these results may be found in the healthy worker selection effect $(32,33)$. Punnett (34) suggested it to be the most likely cause of bias in estimates of the risk for musculoskeletal complaints and concluded that the healthy worker effect acted differentially between high and low exposure groups. Before the start of our study, the (objectively) high exposure group may have become relatively healthy and less susceptible to the development of musculoskeletal complaints, caused by either selection at the start of employment or the dropout of workers susceptible to complaints in the course of early years of employment (35). The selection may have been less pronounced in the medium exposure group, which remained susceptible to complaints. As a result, relatively higher prevalences and prevalence rate ratios can be observed in the medium exposure groups compared with the high exposure groups. Furthermore, the healthy worker effect is suggested to be influenced by gender and socioeconomic status $(32,33,36)$. In our study, an overrepresentation of women (nursing home) was observed in the medium exposure group, and the level of education was identified as a confounder. Both could also have affected the relative health status of the study population at the start of the study and may have attributed to an exposure-response relationship not being found between the observed frequency and duration of pushing and pulling and shoulder complaints.

Exposure was assessed both subjectively and objectively. To obtain relatively unbiased estimates of risk, we used a group-based exposure assessment strategy $(17,37)$. Each individual within each of the exposed or reference groups within each company was assumed to be equally exposed. These subgroups were categorized into three exposure groups. The group strategy reduces precision, but misclassification of exposure and, therefore, attenuation of the risk ratio is usually prevented $(19,37)$. However, differences in the results found between the analyses using self-reported exposure and objectively observed exposure are striking. For self-reported exposure and shoulder complaints a clear doseresponse relationship was observed. But, as already stated, for the observed frequency and duration of pushing and pulling the medium exposure groups were found to have the highest prevalence rate ratios for shoulder complaints. The validity and reliability of the on-site observations was expected to be sufficient. The observers were intensively trained beforehand, and the frequency and duration of the activities were found to be quantifiable with a reasonable level of accuracy $(24,38)$. Therefore, in our study, some groups of workers that were objectively highly exposed classified themselves as subjectively mediumly exposed and some groups of workers that were objectively mediumly exposed classified themselves as subjectively highly exposed. For the objective exposure measures it is expected that, through the application of grouping variables and the methods used for exposure assessment, the misclassification remained nondifferential. If the objectively assessed exposure is considered to be the actual exposure, then the classification methods used for the self-reported exposure may have been differentially biased. Some authors 
have stated that subjects with back pain may overestimate their physical work load relative to those without back pain (2), although others found no evidence for this type of information bias (39). Viikari-Juntura et al (40) reported lower correlations between self-assessed and observed exposure for workers with severe low-back pain in comparison with workers without low-back pain. Workers with severe low-back pain were found to overestimate their exposure. However, in our study, exposure was assessed initially and complaints were recorded at the time of follow-up; in other words, workers who reported complaints overestimated their exposure 1 year earlier. An explanation for this finding may be found in the episodic characteristics of musculoskeletal complaints. It may be that workers who reported complaints at the time of the follow-up experienced (minor) episodes of complaints in the years before the study initiated.

In addition to the assessment of exposure, the assessment of complaints should also be considered when the results are interpreted. Basically, in two succeeding surveys the participants had to recall the occurrence of experiencing complaints in the past year. The reported prevalences may have therefore been inaccurate, but it is expected that such inaccuracy would lead to an underestimation of the prevalence rate ratios (41). Furthermore, given the episodic characteristics of musculoskeletal complaints, we decided not to restrict the analyses to participants without initial complaints, but to adjust for the 12-month prevalence at the beginning of the study. All the participants that had responded at both the beginning and end of the study were included, and, therefore, the selection of participants who were less susceptible to developing complaints was prevented. It may be seriously argued whether the musculoskeletal complaints reported by the participants initially without prior complaints can be considered new events (incidence) $(16,42)$. In this perspective, it may not be appropriate to estimate a risk ratio in terms of relative risk. Therefore, adjusted prevalence rate ratios were calculated using Cox's regression analyses, also because calculating odds ratios by logistic regression is questionable when the prevalence of complaints is relatively high (43-45). It should be noted, however, that the confidence intervals estimated by Cox's regression were expected to be too wide (45).

In all the analyses effect modification by the 12month prevalence at baseline and exposure to pushing and pulling was found to be significant, and, therefore, they were included in the multivariate model. The significantly protective prevalence rate ratios for effect modification (table 4) affect the prevalence rate ratios of both exposure to pushing and pulling and initially reported shoulder complaints. If shoulder complaints were present at the beginning of the study, the present- ed prevalence rate ratios for exposure to pushing and pulling change to values between 0.81 and 1.26 . The prevalence rate ratios for initial shoulder complaints change to values between 2.83 and 4.21 , dependent on the exposure category. Stratified analyses for groups with and without initial shoulder complaints resulted in comparable results.

In conclusion, the findings of the present study suggest a rather strong relationship between pushing and pulling and shoulder complaints. The evidence for a relationship between pushing and pulling and low-back complaints was limited, partly because conservative regression analyses were used. Given the episodic nature of musculoskeletal complaints and the relatively short follow-up period, it is difficult to establish the causality of the relation. The results of our study suggest that the risk of pushing and pulling should be further studied among relatively young and newly contracted workers. Periods with and without complaints should be registered in the course of the follow-up, while, at the same time, the exposure is monitored, as has been suggested by Eisen (42) and Riihimäki (16). Finally, to apply effective ergonomic interventions with respect to pushing and pulling at work, more epidemiologic information is needed of the consequences of aspects of pushing and pulling (eg, handle height and cart weight) $(7,46)$.

\section{Acknowledgments}

The authors would like to thank TNO Work and Employment for advice and the application of the Dutch Musculoskeletal Questionnaire. Josien Coppoolse, Floor Frings, Jitske Groothausen, Jorrit Jansen, Paul Kuijer, Nienke van der Meulen, Tineke Piersma, Annieck Ricken, Marianne Six Dijkstra, Judith Sluiter and Marlies Witjes are gratefully acknowledged for the data collection and processing. Part of the data was collected and processed in co-operation with the Department of Public Health, Erasmus University Rotterdam.

\section{References}

1. Hoogendoorn WE, van Poppel MNM, Bongers PM, Koes BW, Bouter LM. Physical load during work and leisure time as risk factors for back pain. Scand J Work Environ Health 1999;25:387-403.

2. Burdorf A, Sorock G. Positive and negative evidence of risk factors for back disorders [review]. Scand J Work Environ Health 1997;23:243-56.

3. Van der Windt DAWM, Thomas E, Pope DP, De Winter AF, Macfarlane GJ, Bouter LM. Occupational risk factors for shoulder pain: a systematic review. Occup Environ Med 2000;57:433-42. 
4. Van der Heijden GJMG. Shoulder disorders: a state-of-theart review. Baillière's Clini Rheumatol 1999;13:287-309.

5. Baril-Gingras G, Lortie M. The handling of objects other than boxes: univariate analysis of handling techniques in a large transport company. Ergonomics 1995;38:905-25.

6. Bernard BP. Musculoskeletal disorders and workplace factors: a critical review of epidemiological evidence for workrelated musculoskeletal disorders of the neck, upper extremity, and low back. Cincinnati $(\mathrm{OH})$ : National Institute for Occupational Safety and Health, US Department of Health and Human Services, 1997. DHHS report, no 97-141.

7. Hoozemans MJM, van der Beek AJ, Frings-Dresen MHW, van Dijk FJH, Van der Woude LHV. Pushing and pulling in relation to musculoskeletal disorders: a review of risk factors. Ergonomics 1998;41:757-81.

8. Garg A, Moore JS. Epidemiology of low-back pain in industry. Occup Med 1992;7:593-608.

9. Klein BP, Jensen RJ, Sanderson LM. Assessment of workers' compensation claims for back strains/sprains. J Occup Med 1984;26:443-8.

10. Frymoyer JW, Pope MH, Costanza MC, Rosen JC, Goggin JE, Wilder DG. Epidemiologic studies of low-back pain. Spine 1980;5:419-23.

11. Damkot DK, Pope MH, Lord J, Frymoyer JW . The relationship between work history, work environment and low-back pain in men. Spine 1984;9:395-9.

12. Fuortes LJ, Shi Y, Zhang M, Zwerling C, Schootman M. Epidemiology of back injury in university hospital nurses from review of workers' compensation records and a casecontrol survey. J Occup Med 1994;36:1022-6.

13. van der Beek AJ, Frings-Dresen MHW, van Dijk FJH, Kemper HCG, Meijman ThF. Loading and unloading by lorry drivers and musculoskeletal complaints. Int J Ind Ergon 1993;12:13-23.

14. Ozguler A, Leclerc A, Landre M, Pietri-Taleb F, Niedhammer I. Individual and occupational determinants of low back pain according to various definitions of low back pain. J Epidemiol Community Health 2000;54:215-20.

15. Hughes RE, Silverstein BA, Evanoff BA. Risk factors for work-related musculoskeletal disorders in an aluminum smelter. Am J Ind Med 1997;32:66-75.

16. Riihimäki H. Musculoskeletal diseases - a continuing challenge for epidemiologic research. Scand J Work Environ Health 1999;25 suppl 4:31-5.

17. Hoozemans MJM, Burdorf A, van der Beek AJ, FringsDresen MHW, Mathiassen SE. Group-based measurement strategies in exposure assessment explored by bootstrapping. Scand J Work Environ Health 2001;27:125-32.

18. van der Beek AJ, Frings-Dresen MHW. Assessment of mechanical exposure in ergonomic epidemiology. Occup Environ Med 1998;55:291-9.

19. Winkel J, Mathiassen SE. Assessment of physical work load in epidemiologic studies: concepts, issues and operational considerations. Ergonomics 1994;37:979-88.

20. Hildebrandt VH. Prevention of work related musculoskeletal disorders: setting priorities using the standardized Dutch Musculoskeletal Questionnaire [dissertation]. Amsterdam [The Netherlands]: Vrije Universiteit, 2001.

21. Hildebrandt VH, Douwes M. Lichamelijke belasting en arbeid: vragenlijst bewegingsapparaat [Physical load and work: questionnaire on musculoskeletal load and health complaints]. Voorburg [The Netherlands]: Ministerie van Sociale Zaken en Werkgelegenheid, Directoraat-Generaal van de Arbeid, 1991.
22. Hildebrandt VH, Bongers PM, van Dijk FJH, Kemper HCG, Dul J. Dutch musculoskeletal questionnaire: description and basic qualities. Ergonomics 2001;44:1038-55.

23. Frings-Dresen MHW, Kuijer PPFM. The TRAC-system: an observation method for analysing work demands at the workplace. Saf Sci 1995;21:163-5.

24. van der Beek AJ, Van Gaalen LC, Frings-Dresen MHW. Working postures and activities of lorry drivers: a reliability study of on-site observation and recording on a pocket computer. Appl Ergon 1992;23:331-6.

25. Dempsey PG, Burdorf A, Webster BS. The influence of personal variables on work-related low-back disorders and implications for future research. J Occup Environ Med 1997; 39:748-59.

26. Kuiper JI, Burdorf A, Verbeek JHAM, Frings-Dresen MHW, van der Beek AJ, Viikari-Juntura E. Epidemiological evidence on manual materials handling as a risk factor for back disorders: a systematic review. Int J Ind Ergon 1999;24:389_ 404.

27. Sluiter JK, Rest KM, Frings-Dresen MHW. Criteria document for evaluation of the work-relatedness of upper extremity musculoskeletal disorders. Scand J Work Environ Health 2001;27 suppl 1:1-102.

28. Bongers PM, de Winter CR, Kompier MAJ, Hildebrandt VH. Psychosocial factors at work and musculoskeletal disease. Scand J Work Environ Health 1993;19:297-312.

29. Van Veldhoven M. Psychosocial job demands and workstress [dissertation]. Groningen [The Netherlands]: University of Groningen, 1996.

30. Kuorinka IAA, Jonsson B, Kilbom Å, Vinterberg H, BieringSorensen F, Andersson GBJ, et al. Standardised Nordic questionnaires for the analysis of musculoskeletal symptoms. Appl Ergon 1987;18:233-7.

31. De Graaf R, Bijl RV, Smit F, Ravelli A, Volleberh WAM. Psychiatric and sociodemographic predictors of attrition in a longitudinal study. Am J Epidemiol 2000;152:1039-47.

32. Li C-Y, Sung F-C. A review of the healthy worker effect in occupational epidemiology. Occup Med 1999;49:225-9.

33. Choi BCK. Definition, sources, magnitude, effect modifiers, and strategies of reduction of the healthy worker effect. $\mathrm{J}$ Occup Med 1992;34:979-88.

34. Punnett L. Adjusting for the healthy worker selection effect in cross-sectional studies. Int J Epidemiol 1996;25:1068-76.

35. De Zwart BCH, Broersen JPJ, van der Beek AJ, FringsDresen MHW, van Dijk FJH. Selection related to musculoskeletal complaints among employees. Occup Environ Med 1997;54:800-6.

36. Lea CS, Hertz-Picciotto I, Andersen A, Chang-Claude J, Olsen JH, Pesatori AC, et al. Gender differences in the healthy worker effect among synthetic vitreous fiber workers. Am J Epidemiol 1999;150:1099-106.

37. Seixas NS, Sheppard L. Maximizing accuracy and precision using individual and grouped exposure assessments. Scand J Work Environ Health 1996;22:94-101.

38. Kilbom $\AA$. Assessment of physical exposure in relation to work-related musculoskeletal disorders - what information can be obtained from systematic observations? Scand J Work Environ Health 1994;20 special issue:30-45.

39. Toomingas A, Alfredsson L, Kilbom A. Possible bias from rating behavior when subjects rate both exposure and outcome. Scand J Work Environ Health 1997;23:370-7.

40. Viikari-Juntura E, Rauas S, Martikainen R, Kuosma E, Riihimäki H, Takala E-P, et al. Validity of self-reported physical work load in epidemiologic studies on musculoskeletal 
disorders. Scand J Work Environ Health 1996;22:251-9.

41. Altman DG. Practical statistics for medical research. London: Chapman \& Hall, 1991.

42. Eisen EA. Methodology for analyzing episodic events. Scand J Work Environ Health 1999;25 suppl 4:36-42.

43. Thompson ML, Myers JE, Kriebel D. Prevalence odds ratio or prevalence ratio in the analysis of cross sectional data: what is to be done? Occup Environ Med 1998;55:272-7.

44. Nurminen M. To use or not to use the odds ratio in epidemiologic analyses? Eur J Epidemiol 1995;11:365-71.
45. Skov T, Deddens J, Petersen MR, Endahl L. Prevalence proportion ratios: estimation and hypothesis testing. Int $\mathrm{J}$ Epidemiol 1998;27:91-5.

46. van der Beek AJ, Hoozemans MJM, Frings-Dresen MHW, Burdorf A. Assessment of exposure to pushing and pulling in epidemiological field studies: an overview of methods, exposure measures, and measurement strategies. Int J Ind Ergon 1999;24:417-29.

Received for publication: 31 May 2001

\section{Appendix}

Detailed company-level information on the response to the questionnaire survey, number of male and female participants, prevalences (\%) of low-back and shoulder complaints in the baseline and follow-up surveys, distribution of companies over exposure categories $(1=$ reference group, $2=$ medium exposure group, $3=$ high exposure group $)$, and the actual mean frequency (times per workday) and duration (seconds per workday) of manual materials handling

\begin{tabular}{|c|c|c|c|c|c|c|c|c|c|}
\hline & \multirow{2}{*}{$\begin{array}{l}\text { Population } \\
\text { (N) }\end{array}$} & \multicolumn{2}{|c|}{ Response } & \multirow{2}{*}{$\begin{array}{l}\text { Men } \\
(\mathrm{N})\end{array}$} & \multirow{2}{*}{$\begin{array}{l}\text { Women } \\
\qquad(\mathrm{N})\end{array}$} & \multicolumn{2}{|c|}{$\begin{array}{l}\text { Prevalence of low-back } \\
\text { complaints (\%) }\end{array}$} & \multicolumn{2}{|c|}{$\begin{array}{l}\text { Prevalence of shoulder } \\
\text { complaints (\%) }\end{array}$} \\
\hline & & Baseline & Follow-up & & & Baseline & Follow-up & Baseline & Follow-up \\
\hline \multicolumn{10}{|l|}{ Rail catering company } \\
\hline Exposed group & 97 & 69 & 52 & 41 & 11 & 49 & 54 & 45 & 45 \\
\hline Reference group & 33 & 22 & 10 & 6 & 4 & 70 & 50 & 20 & 20 \\
\hline \multicolumn{10}{|l|}{ Hower auction 1} \\
\hline Exposed group 1 & 62 & 38 & 23 & 23 & - & 61 & 65 & 70 & 70 \\
\hline Exposed group 2 & 15 & 10 & 7 & 5 & 2 & 43 & 57 & 71 & 43 \\
\hline Reference group & 56 & 48 & 34 & 32 & 2 & 38 & 32 & 15 & 12 \\
\hline \multicolumn{10}{|l|}{ Hower auction 2} \\
\hline Exposed group & 74 & 60 & 52 & 45 & 7 & 32 & 37 & 18 & 14 \\
\hline Reference group & 41 & 35 & 31 & 13 & 18 & 42 & 26 & 39 & 30 \\
\hline \multicolumn{10}{|l|}{ Nursing home } \\
\hline Exposed group & 136 & 121 & 109 & 7 & 102 & 61 & 60 & 38 & 43 \\
\hline Reference group & 109 & 85 & 65 & 14 & 51 & 54 & 52 & 27 & 22 \\
\hline \multicolumn{10}{|l|}{ Postal distribution center } \\
\hline Exposed group & 112 & 68 & 34 & 34 & 0 & 35 & 44 & 24 & 35 \\
\hline $\begin{array}{l}\text { Refuse collection companies } \\
\text { Exposed group }\end{array}$ & 94 & 73 & 42 & 42 & 0 & 42 & 44 & 22 & 17 \\
\hline Total & 829 & 629 & 459 & 262 & 197 & & & & \\
\hline
\end{tabular}




\begin{tabular}{|c|c|c|c|c|c|c|c|c|c|}
\hline & \multicolumn{3}{|c|}{ Exposure categories } & \multicolumn{2}{|l|}{ Pushing } & \multicolumn{2}{|l|}{ Pulling } & \multirow{2}{*}{$\begin{array}{l}\text { Lifting } \\
\text { frequency } \\
\text { (mean) }\end{array}$} & \multirow{2}{*}{$\begin{array}{l}\text { Carrying } \\
\text { frequency } \\
\text { (mean) }\end{array}$} \\
\hline & $\begin{array}{l}\text { Self-reported } \\
\text { exposure }\end{array}$ & $\begin{array}{l}\text { Observed } \\
\text { frequency }\end{array}$ & $\begin{array}{l}\text { Observed } \\
\text { duration }\end{array}$ & $\begin{array}{l}\text { Frequency } \\
\text { (mean) }\end{array}$ & $\begin{array}{l}\text { Duration } \\
\text { (mean) }\end{array}$ & $\begin{array}{l}\text { Frequency } \\
\text { (mean) }\end{array}$ & $\begin{array}{l}\text { Duration } \\
\text { (mean) }\end{array}$ & & \\
\hline \multicolumn{10}{|c|}{ Rail catering company } \\
\hline Exposed group & 3 & 2 & 3 & 196 & 4360 & 38 & 335 & 7 & 5 \\
\hline Reference group & 1 & 1 & 1 & - & - & - & - & - & - \\
\hline \multicolumn{10}{|l|}{ Hower auction 1} \\
\hline Exposed group 1 & 3 & 3 & 2 & 56 & 280 & 620 & 1409 & 2 & 58 \\
\hline Exposed group 2 & 3 & 3 & 3 & 122 & 718 & 342 & 2846 & 13 & 154 \\
\hline Reference group & 1 & 1 & 1 & 1 & 14 & 1 & 9 & 6 & 5 \\
\hline \multicolumn{10}{|l|}{ Hower auction 2} \\
\hline Exposed group & 2 & 3 & 3 & 159 & 838 & 455 & 2684 & 88 & 11 \\
\hline Reference group & 1 & 1 & 1 & 10 & 92 & 11 & 92 & 17 & 3 \\
\hline \multicolumn{10}{|l|}{ Nursing home } \\
\hline Exposed group & 2 & 2 & 2 & 57 & 557 & 31 & 226 & 22 & 15 \\
\hline Reference group & 1 & 1 & 1 & 11 & 142 & 15 & 39 & 3 & 7 \\
\hline \multicolumn{10}{|c|}{ Postal distribution center } \\
\hline Exposed group & 3 & 3 & 3 & 342 & 1928 & 400 & 2422 & 218 & 9 \\
\hline \multicolumn{10}{|c|}{ Refuse collection companices } \\
\hline Exposed group & 2 & 3 & 2 & 446 & 1146 & 536 & 1453 & 9 & 1 \\
\hline
\end{tabular}

